năm $(17,9 \%)$. Kent và cộng sự [9] đã cho thấy những bệnh nhân động kinh trong thời gian dài tỉ lệ bị suy giảm trí nhớ cao hơn cũng như điểm số trong các thang điểm đánh giá chức năng trí nhớ cũng thấp hơn. Cả tần suất xuất hiện cớn và thời gian mắc bệnh đều có liên quan tới chức năng trí nhớ. Tần suất cơn càng dày, thời gian mắc bệnh càng lâu thì chức năng cao cấp của não càng dễ bị ảnh hưởng gây suy giảm chức năng trí nhớ, rõ ràng cần có sự can thiệp điều trị tích cực với bệnh nhân động kinh để giảm thiểu ảnh hưởng xấu lên cuộc sống của họ.

\section{KẾT LUÂ̂N}

Bệnh động kinh là bệnh lý mạn tính của não với tỉ lệ mắc tương đối cao. Bệnh ngoài gây xuất hiện cởn động kinh còn gây ảnh hưởng đên chức năng cao cấp của não, trong đó có gây suy giảm trí nhớ, làm giảm chất lượng cuộc sống của người bệnh. Nhiều yếu tố của bệnh động kinh của liên quan đến suy giảm trí nhớ, đặc biệt là tần suất xuất hiện cơn và thời gian mắc bệnh. Do đó cần có sự quan tâm lớn hơn từ các bác sĩ lâm sàng, góp phần xây dựng chiến lược chăm sóc và điều trị tốt hơn cho bệnh nhân.

\section{TÀl LIẸU THAM KHẢO}

1. Fisher R.S., van Emde Boas W., Blume W. và cộng sự. (2005). Epileptic seizures and epilepsy: definitions proposed by the International League Against Epilepsy (ILAE) and the International Bureau for Epilepsy (IBE). Epilepsia, 46(4), 470-472.

2. Fisher R.S., Acevedo C., Arzimanoglou A. và cộng sự. (2014). ILAE official report: a practical clinical definition of epilepsy. Epilepsia, 55(4), 475-482.

3. Lê Thế Phi (2018), Đánh giá ảnh hưởng của thuốc phenobarbital lến chức năng nhận thức trên bênh nhân trưởng thành mắc đông kỉnh cơn lớn. Luận văn Thạc sĩ Y học, Trường Đại học Y Hà Nội.

4. Hồ Anh Thủy (2011), Nghiên cứu môt số đặc điểm về rối loạn nhận thức trên bệnh nhân đông kinh người trưởng thành được điều trị bắng phenobarbital. Luận văn tốt nghiệp Bác sỹ chuyên khoa cấp II, Trường Đại học Y Hà Nội, Hà Nội.

5. Beghi E. (2020). The Epidemiology of Epilepsy. NED, 54(2), 185-191.

6. Kotsopoulos I., de Krom M., Kessels F. và cộng sự. (2005). Incidence of epilepsy and predictive factors of epileptic and non-epileptic seizures. Seizure, 14(3), 175-182.

7. Marques C.M., Caboclo L.O.S.F., da Silva T.I. và cộng sự. (2007). Cognitive decline in temporal lobe epilepsy due to unilateral hippocampal sclerosis. Epilepsy Behav, 10(3), 477-485.

8. Wang L., Chen S., Liu C. và cộng sứ. (2019). Factors for cognitive impairment in adult epileptic patients. Brain Behav, 10(1), e01475.

9. Kent G.P., Schefft B.K., Howe S.R. và cộng sự. (2006). The effects of duration of intractable epilepsy on memory function. Epilepsy Behav $9(3), 469-477$.

\title{
SỬ DƯNG TÍNH TOÁN DFT KẾT HỢP VỚI THỰC NGHIỆM ĐỂ XÁC ĐINH CÁC ĐĂC TÍNH CỦA BENZOYL PEROXID
}

\section{TÓM TẮT}

Hoạt chất benzoyl peroxid được sử dụng bôi ngoài da để điều trị mụn trứng cá, tiêu sừng và chống viêm. Kết quả quang phổ Raman, IR thu được từ thực nghiệm được so sánh với các tính toán lượng tử Gaussian với DFT/B3LYP kết hớp với các tiêu chuẩn 6$311+G(d, p)$. Các đặc tính lý hóa và đặc tính dược lực của phân tử hữu cơ benzoyl peroxide đã được phẩn tích bằng quang phổ IR, Raman. Vị trí số sóng đặc trưng của các liên kết, các nhóm trong benzoyl peroxid đều bị lệch nhiêu so với vị trí dải bước sóng thông thường. Phân tích điện Mulliken cho thấy các đám mây điện tử dẫn hướng qua carbon liên kết với

*Trường Đại học Dược Hà Nội

Chịu trách nhiệm chính: Nguyễn Đức Thiện

Email: thiennd@hup.edu.vn

Ngày nhân bài: 3.8.2021

Ngày phản biện khoa học: 29.9.2021

Ngày duyệt bài: 6.10.2021

\section{Nguyễn Đức Thiện*, Nguyễn Thị Mỹ Linh*,} Lê Thị Phương Anh*, Điêu Diễm Quỳnh*

nhóm peroxide của phân tử trên cùng của vòng benzen. Độ âm điện và thế hóa học của benzoyl peroxide có cùng đồ lớn là 4,72955 , cho các liên kết hóa học chặt chẽ và độ bền liên kết của electron cao.

Tư khóa: benzoyl peroxide, phổ Raman, phổ IR, phân bố điện tích Mulliken, DFT.

\section{SUMMARY \\ USING DFT CALCULATIONS COMBINED WITH EXPERIMENTAL DATA TO DETERMINE THE CHARACTERISTICS OF BENZOYL PEROXIDE}

The compound benzoyl peroxide is used topically to treat acne, keratosis, and anti-inflammatory. The results of Raman and IR spectroscopy obtained in the experiment were compared with the Gaussian quantum calculations with DFT/B3LYP the basis sets $6-311 G+(d, p)$. The pharmacodynamic activity of the organic molecule benzoyl peroxide was analyzed by IR, Raman spectroscopy. The wavenumber positions of the bond in the benzoyl peroxide are all deviated from below the usual wavelength range. Mulliken 
electrical analysis showed that electron clouds guided through the carbon bonded to the peroxide group of the top molecule of the benzene ring. The electronegativity and chemical potential of benzoyl peroxide are of the order of 472955 , indicating tight chemical bonds and high electron bonding strength.

Keywords: benzoyl peroxide, Raman spectra, IR spectra, Mulliken charge distribution, DFT.

\section{I. ĐẶT VẤN ĐỀ}

Benzoyl peroxide $\left(\mathrm{C}_{14} \mathrm{H}_{10} \mathrm{O}_{4}\right)$ (BPO) là một hợp chất hữu cơ thuộc họ peroxide và nó gồm hai vòng benzen được ghép bởi một nhóm peroxide. BPO là một chất rắn kết tinh, có cấu trúc tinh thể trực thoi, hòa tan trong ethanol, ether và độ hòa

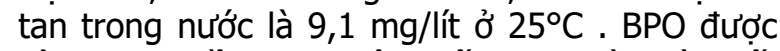
sử dụng nhiều như một chất khơi mào và chất xúc tác trong công nghiệp bảo quản thực phẩm. BPO là một dược chất quan trọng để điều trị mụn trứng cá, do hoạt động kháng khuẩn của nó chống lại vi khuẩn Propionibacterium acnes [1]. BPO đã được sử dụng nhiều dạng thuốc bôi ngoài da để điều trị mụn trứng cá ở trên thế giới [2], hơn thế nữa nó còn có tác dụng tiêu sừng/tiêu mụn và chống viêm [3]. Tổng hợp nghiên cứu gần đây chỉ ra rằng $\mathrm{BPO}$ là loại thuốc an toàn, có ứng dụng kháng sinh và chống viêm quan trọng. Tuy nhiên, BPO vẫn cần được nghiên cứu về đặc tính phân tử, đặc biệt là đặc tính dược lực. Do vậy, bài nghiên cứu này sử dụng dữ liệu quang phổ và kết quả tính toán lượng tử để góp phần làm rõ hơn đặc tính của BPO.

\section{NGUYÊN UỆU VÀ PHƯƠNG PHÁP NGHIÊN CỨU}

a. Nguyên liệu. Benzoyl peroxid $\mathrm{C}_{14} \mathrm{H}_{10} \mathrm{O}_{4}$ (Trung Quốc), số lô 20080703, độ tinh khiết $\geq$ 98,0\% được sử dụng trực tiếp để đo phổ Raman, hồng ngoại mà không qua bất kỳ xử lý nào.

b. Phương pháp nghiên cứu. Hình thái, cấu trúc điện tữ, tính chất nhiệt động, tần số dao động IR, Raman của $\mathrm{BPO}$ và orbital phân tử (MO) của phân tử BPO được tối ưu hóa và tính toán bằng phương pháp lý thuyết phiếm hàm mật độ (DFT) sử dụng ba thông số trao đổi hàm loại Beckes (B3) kết hợp với phép tương quan hàm số Lee-Yang-Parr (LYP) và cài đặt tiêu chuẩn $6-311 \mathrm{G}+(\mathrm{d}, \mathrm{p})$, trên máy tính sử dụng phần mềm Gaussian 09W và Gaussview 5.0 ([4], [5]). Chương trình chạy để tối ưu hóa bằng HF/3-21G cho hội tụ và để tìm initial force constant. Tiếp tục chạy B3LYP/6-311G+(d) với $\mathrm{T}=298 \mathrm{~K}$, không cài đặt \%nproc và \%mem.
Việc gán điện tích Mulliken trên các phần khác nhau của hợp chất đã được tính toán và được làm sáng tỏ một cách có chủ đích để xác định đặc tính và hoạt tính dược phẩm của hợp chất. Bằng phương pháp DFT với bộ tiêu chuẩn 6-311G+(d, p) các thông số đặc trưng lý hóa như độ âm điện, thế hóa học, độ rộng vùng cấm năng lượng được xác định, qua đó lý giải đặc tính động lực học của BPO.

Kết quả đo thực nghiệm của BPO: phổ IR đo bằng Shimadru (số sóng: $400 \div 4000 \mathrm{~cm}^{-1}$ ) tại Khoa Hóa, Trường Đại học Khoa học Tự nhiên; phổ Raman đo bằng Horiba LabRAM HR Evolution (bước sóng kích thích: $785 \mathrm{~nm}$; dịch chuyển Raman: $176 \div 2000 \mathrm{~cm}^{-1}$ ) tại Khoa Vật lý, Trường Đại học Sư phạm Hà Nội.

\section{KẾT QUẢ NGHIÊN CỨU VÀ BÀN LUÂ̂N}

3.1. Hình thái phân tử. Một phân tử $B P O$ trong pha khí được tối ưu hóa có các nguyên tử và được đánh số như hình 1 . Phân tử $\mathrm{BPO}$ có hai vòng benzen được kết nối với nhau bằng hai nhóm peroxide. Để momen điện hóa bằng nhau thì hai vòng benzen đặt vuông góc nhau, liên kết cộng hóa trị giữa $\mathrm{O} 1$ và $\mathrm{O} 2$ được coi là liên kết đồng hóa trị. Theo quan điểm về nhóm điểm thì BPO thuộc nhóm điểm $C_{2 v}$. Các đặc tính phân tử của BPO được nghiên cứu tính toán cho ra các thông số độ hấp thụ riêng thu được bằng phần mềm HyperChem 8.0.6 được chỉ ra trong bảng 1 .

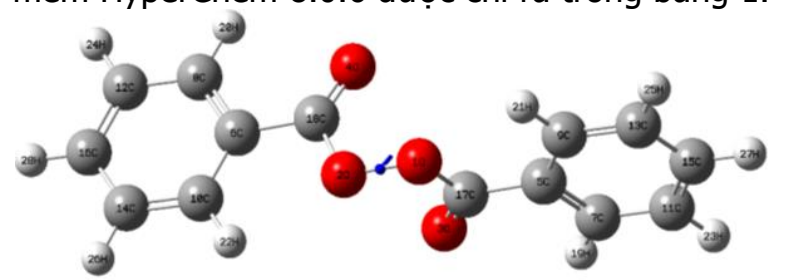

Hình 1. Câu trúc phân tử BPO được tôi ưu hóa.

Bảng 1. Thông số vật lý của Benzoyl peroxid

\begin{tabular}{|c|c|}
\hline Thông số & Giá trị \\
\hline Số lượng nhường liên kết hidro & 0 \\
\hline Số lượng nhận liên kết hidro & 4 \\
\hline Số lượng liên kết xoay & 5 \\
\hline Diện tích bề mặt phân cực theo topo & $52,6 \AA^{2}$ \\
\hline Khối lượng chính xác & 242,058 \\
$\mathrm{~g} / \mathrm{mol}$ \\
\hline Số nguyên tử nặng & 18 \\
\hline Số lượng liên kết đồng hóa trị & 1 \\
\hline
\end{tabular}

Các thông số hình học được tính toán tối ưu hóa về độ dài, góc các liên kết trong phân tử đã được trình bày trong bảng 2 .

\section{Bảng 2. Các thông số hình học được tôi ưu hóa cho BPO được tính toán ở HF/DFT (B3LYP) với bộ cơ sớ 6-311G+(d, p).}


VIETNAM MEDICAL JOURNAL N02 - OCTOBER - 2021

\begin{tabular}{|c|c|c|c|c|c|c|c|}
\hline \begin{tabular}{|c|c|} 
Dộ dài liên kế \\
$(\AA)$
\end{tabular} & HF & $\begin{array}{l}\text { B3LYP 6- } \\
\text { 311G+(d,p }\end{array}$ & B3PW91 & $\begin{array}{l}\text { Đô dài liên } \\
\text { kết }(\AA)\end{array}$ & HF & \begin{tabular}{|c|} 
B3LYP 6- \\
311G+(d,p)
\end{tabular} & B3PW91 \\
\hline \begin{tabular}{|l|}
$\mathrm{O} 1-\mathrm{O} 2$ \\
\end{tabular} & 1,4583 & 1,4583 & 1,4583 & C9-C13 & 1,3949 & 1,3948 & 1,3949 \\
\hline O1-C17 & 1,3595 & 1,37 & 1,3595 & $\mathrm{C} 9-\mathrm{H} 21$ & 1,0859 & 1,0869 & 1,0859 \\
\hline $\mathrm{O} 2-\mathrm{C} 18$ & 1,37 & 1,3595 & 1,37 & C10-C14 & 1,3948 & 1,3949 & 1,3948 \\
\hline O3-C17 & 1,2212 & 1,2379 & 1,2212 & $\mathrm{C} 10-\mathrm{H} 22$ & 1,0869 & 1,0859 & 1,0869 \\
\hline O4-C18 & 1,2379 & 1,2212 & 1,2379 & C11-C15 & 1,3948 & 1,3948 & 1,3948 \\
\hline $\mathrm{C} 5-\mathrm{C} 7$ & 1,38 & 1,3948 & 1,38 & $\mathrm{C} 11-\mathrm{H} 23$ & 1,0862 & 1,0861 & 1,0862 \\
\hline C5-C9 & 1,38 & 1,3949 & 1,38 & C12-C16 & 1,3948 & 1,3948 & 1,3948 \\
\hline C5-C17 & 1,4843 & 1,4542 & 1,4843 & $\mathrm{C} 12-\mathrm{H} 24$ & 1,0861 & 1,0862 & 1,0861 \\
\hline $\mathrm{C} 6-\mathrm{C} 8$ & 1,3948 & 1,38 & 1,3948 & C13-C15 & 1,3949 & 1,3948 & 1,3949 \\
\hline $\mathrm{C} 6-\mathrm{C} 10$ & 1,3949 & 1,38 & 1,3949 & $\mathrm{C} 13-\mathrm{H} 25$ & 1,0862 & 1,0861 & 1,0862 \\
\hline C6-C18 & 1,4542 & 1,4843 & 1,4542 & C14-C16 & 1,3948 & & 1,3948 \\
\hline C7-C11 & 1,4542 & 1,3948 & 1,3948 & $\mathrm{C} 14-\mathrm{H} 26$ & 1,0861 & 1,0862 & 1,0861 \\
\hline C7-H19 & 1,086 & 1,0869 & 1,086 & $\mathrm{C} 15-\mathrm{H} 27$ & 1,0861 & 1,0861 & 1,0861 \\
\hline $\mathrm{C} 8-\mathrm{C} 12$ & 1,3948 & 1,3948 & 1,3948 & $\mathrm{C} 16-\mathrm{H} 28$ & & & \\
\hline $\mathrm{C} 8-\mathrm{H} 20$ & 1,0869 & 1,086 & 1,0869 & & & & \\
\hline $\begin{array}{c}\text { Góc giữa } \\
\text { liên kết (độ) }\end{array}$ & HF & $\begin{array}{c}\text { B3LYP 6- } \\
311 \mathrm{G}+(\mathrm{d}, \mathrm{p}\end{array}$ & B3PW91 & $\begin{array}{c}\text { Góc giữa liên } \\
\text { kết (độ) }\end{array}$ & HF & \begin{tabular}{|c|} 
B3LYP 6- \\
$311 G+(d, p)$
\end{tabular} & B3PW91 \\
\hline $\mathrm{O} 2-\mathrm{O} 1-\mathrm{C} 17$ & 106,7359 & 107,9146 & 106,7359 & $\mathrm{C} 14-\mathrm{C} 10-\mathrm{H} 22$ & 118,335 & 118,5089 & \begin{tabular}{|l|}
118,335 \\
\end{tabular} \\
\hline $\mathrm{O} 1-\mathrm{O} 2-\mathrm{C} 18$ & 107,9146 & 106,7359 & 107,9146 & C7-C11-C15 & 119,9992 & ,998 & 119,9992 \\
\hline C7-C5-C9 & 122,1647 & 119,9931 & 122,1647 & $\mathrm{C} 7-\mathrm{C} 11-\mathrm{H} 23$ & 120,0082 & 120,0119 & 120,0082 \\
\hline $\mathrm{C} 7-\mathrm{C} 5-\mathrm{C} 17$ & 118,9299 & 120,0153 & 118,9299 & $\mathrm{C} 15-\mathrm{C} 11-\mathrm{H} 23$ & 119,9926 & 119,99 & 119,9926 \\
\hline C9-C5-C17 & 118,9053 & 119,9916 & 118,9053 & C8-C12-C16 & 119,998 & 119,9992 & 119,998 \\
\hline C8-C6-C10 & 119,9931 & 122,1647 & 119,9931 & $\mathrm{C} 8-\mathrm{C} 12-\mathrm{H} 24$ & 120,0119 & 120,0082 & 120,0119 \\
\hline C8-C6-C18 & 120,0153 & 118,9299 & 120,0153 & $\mathrm{C} 16-\mathrm{C} 12-\mathrm{H} 24$ & 119,99 & 119,9926 & 119,99 \\
\hline C10-C6-C18 & 119,9916 & 118,9053 & 119,9916 & C9-C13-C15 & 119,9985 & 119,9968 & 119,9985 \\
\hline C5-C7-C11 & 118,9204 & 120,0055 & 118,9204 & $\mathrm{C} 9-\mathrm{C} 13-\mathrm{H} 25$ & 120,021 & 120,0226 & 120,021 \\
\hline $\mathrm{C} 5-\mathrm{C} 7-\mathrm{H} 19$ & 121,7623 & 120,9398 & 121,7623 & $\mathrm{C} 15-\mathrm{C} 13-\mathrm{H} 25$ & 119,9803 & 119,9803 & 119,9803 \\
\hline $\mathrm{C} 11-\mathrm{C} 7-\mathrm{H} 19$ & 119,317 & 119,9544 & 119,317 & $\mathrm{C} 10-\mathrm{C} 14-\mathrm{C} 16$ & 119,9968 & 119,9985 & 119,9968 \\
\hline C6-C8-C12 & 120,0055 & 118,9204 & 120,0055 & $\mathrm{C} 10-\mathrm{C} 14-\mathrm{H} 26$ & 120,0226 & 120,021 & 120,0226 \\
\hline $\mathrm{C} 6-\mathrm{C} 8-\mathrm{H} 20$ & 120,9398 & 121,7623 & 120,9398 & $\mathrm{C} 16-\mathrm{C} 14-\mathrm{H} 26$ & 119,9803 & 119,9803 & 119,9803 \\
\hline $\mathrm{C} 12-\mathrm{C} 8-\mathrm{H} 20$ & 119,0544 & 119,317 & 119,0544 & C11-C15-C13 & 120,0014 & 120,0031 & 120,0014 \\
\hline C5-C9-C13 & 118,9158 & 120,0035 & 118,9158 & $\mathrm{C} 11-\mathrm{C} 15-\mathrm{H} 27$ & 120,001 & 120,0014 & 120,001 \\
\hline $\mathrm{C} 5-\mathrm{C} 9-\mathrm{H} 21$ & 122,5693 & 121,6572 & 122,5693 & $\mathrm{C} 13-\mathrm{C} 15-\mathrm{H} 27$ & 119,9976 & 119,9955 & 119,9976 \\
\hline $\mathrm{C} 13-\mathrm{C} 9-\mathrm{H} 21$ & 118,5089 & 118,335 & 118,5089 & C12-C16-C14 & 120,0031 & 120,0014 & 120,0031 \\
\hline C6-C10-C14 & 120,0035 & 118,9158 & 120,0035 & $\mathrm{C} 12-\mathrm{C} 16-\mathrm{H} 28$ & 120,0014 & 120,001 & 120,0014 \\
\hline $\mathrm{C} 6-\mathrm{C} 10-\mathrm{H} 22$ & 121,6572 & 122,5693 & 121,6572 & $\mathrm{C} 14-\mathrm{C} 16-\mathrm{H} 28$ & 119,9955 & 119,9976 & 119,9955 \\
\hline
\end{tabular}

\subsection{Phân bố điên tích Mulliken}

Bảng 3. Điện tích theo phân bố Mulliken các nguyên tử trong phân tử BPO với cài đặt cơ sở 6-311G+ $(d, p)$.

\begin{tabular}{|c|c|c|c|c|c|c|c|}
\hline Nguyên tử & 01 & 02 & 03 & 04 & C5 & C6 & C7 \\
\hline Điện tích (e) & 0,09940 & 0,10891 & $-0,23514$ & $-0,22845$ & 0,99083 & 0,97643 & $-0,35788$ \\
\hline & C8 & C9 & C10 & C11 & C12 & C1 & C14 \\
\hline Điện & $-0,34540$ & $-0,13073$ & $-0,11977$ & $-0,20319$ & $-0,21576$ & $-0,25799$ & $-0,27422$ \\
\hline Nguy & C15 & C16 & C17 & C18 & H19 & H2O & H21 \\
\hline & $-0,1$ & & $-0,5$ & 0,4 & & & 0115271 \\
\hline & $\mathrm{H}_{2}$ & & $\mathrm{H2}$ & $\mathrm{H}_{2}$ & $\mathbf{H}$ & H27 & H28 \\
\hline Điện tích (e) & 0,13605 & 0,13605 & 0,13441 & 0,13475 & 0,13311 & 0,13524 & 0,13362 \\
\hline
\end{tabular}

Theo phân bố Mulliken thì điện tích các nguyên tử trong phân tử BPO với cài đăt cơ sở 6$311 \mathrm{G}+(\mathrm{d}, \mathrm{p})$ ở bảng 3 . Theo lý thuyết DFT thì sự phân bố điện tích giữa các obitan phân tử thể hiện sự chuyển đổi năng lượng để tạo ra các đặc tính phân tử như tính chất vật lý, hóa học. Sự tiêu thụ năng lượng trong quá trình dao động cho biết sự định hướng của thế điện tử trong các phần khác nhau của hợp chất. Thông thường, đám mây điện tử được định hướng về phía của 
nhóm peroxide vì nó chứa 4 oxy. Tại đây, các đám mây điện tử tập trung trực tiểp trên các nguyên tử carbon của vòng benzen và bắc cầu qua carbon liên kết với nhóm peroxide của phân tử trên cùng của vòng benzen. Như vậy đám mây điện tử đã bao trùm toàn khối phân tử BPO và làm cho $\mathrm{BPO}$ có hoạt tính mạnh đồng nhất.

Do sự định hướng, các carbon của các nhóm peroxide trở nên trung tính, trong khi đám mây electron vẫn tập trung ở 03, 04 và có liên kết n để duy trì trạng thái cân bằng hóa học. Ngoài ra, các liên kết lưỡng cực mạnh $(\mathrm{C}=0)$ được tạo ra đối xứng trên các nhóm peroxide. Phân bố điện tích âm xuất hiện ở cả hai đầu và nó bị suy giảm vùng giữa phân tử tập trung ở nhóm peroxide, do đó đặc tính thuốc không phân biệt đầu hay cuối phân tử BPO.

Bảng 3 cho thấy khối lượng proton ở trong các nguyên tử carbon của cả hai vòng benzen nhiều hơn các nguyên tử khác, điều này là vì các điện tích dao động qua lại thông qua nhóm peroxide. Cách thức định hướng điện tích không đối xứng này gây ra hiệu ứng chổng viêm của BPO [6]. Điện tích không đối xứng và sự phân chia trong BPO được thể hiện rõ ràng qua màu sắc trong hình 3.

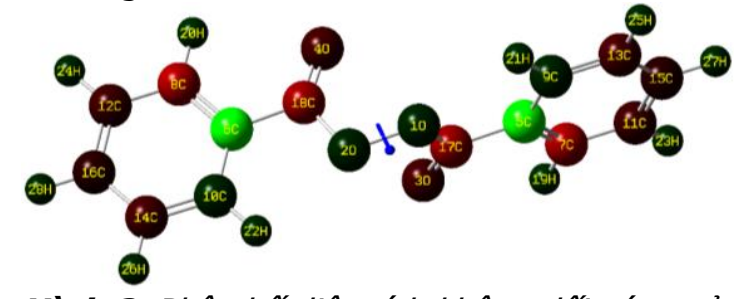

Hinh 3. Phân bố điện tích không đối xứng của nguyên tử trong phân tử BPO theo Mulliken

3.3. Phổ IR, Raman và gán dao động

Phổ IR, Raman của $B P O$ theo DFT và thực nghiệm đo được ở hình 4 và hình 5.

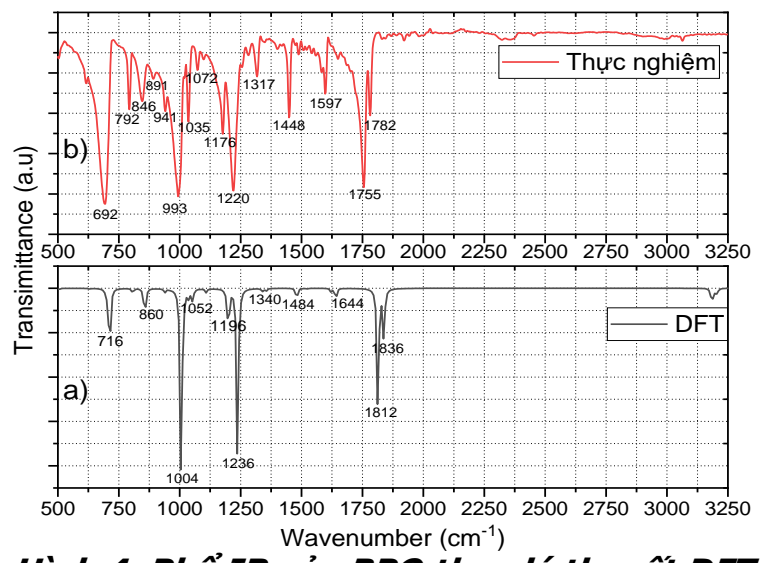

Hình 4. Phổ IR của BPO theo lý thuyêt DFT a) và thực nghiệm b).
Từ cấu trúc tối ưu phân tử BPO ở hình 1 cho thấy các nguyên tử thuộc nhiều mặt phẳng khác nhau, phân tử này có 28 nguyên tử nên có 78 dao động (53 dao động trong mặt phẳng và 25 dao động ngoài mặt phẳng). Kết hợp với kết quả thực nghiệm đo được, chúng tôi đưa ra một số đỉnh phổ đặc trưng rõ ràng trong phân tử BPO và gán các kiểu dao động tươnng ứng với các liên kết (Bảng 4). Các dao động của các liên kết trong BPO chủ yếu là 3 kiểu dao động: $\mathrm{C}-\mathrm{H}, \mathrm{CC}$ trong hai vòng benzen và nhóm peroxid.

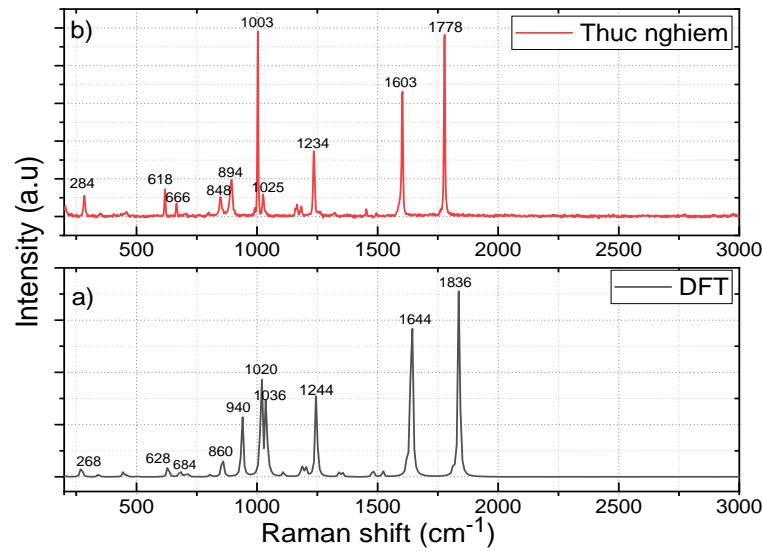

Hình 5. Phổ Raman của BPO theo lý thuyết DFT a) và thức nghiêtm b).

Do nhóm peroxid liên kết giữa hai vòng benzen nên chỉ còn có 10 liên kết $\mathrm{C}-\mathrm{H}$, do đó có 20 dao động uốn sẽ được quan sát thấy. 10 dao động uốn trong mặt phẳng $\mathrm{C}-\mathrm{H}$ được tìm thấy từ $1072-840 \mathrm{~cm}^{-1}$. 10 dao động uốn cong ngoài mặt phẳng $\mathrm{C}-\mathrm{H}$ được quan sát tại $840-500 \mathrm{~cm}^{-1}$. Thông thường, hai dải dao động uốn khác nhau này được xác định trong vùng lân lượt là $1300-$ $1000 \mathrm{~cm}^{-1}$ và $1000-750 \mathrm{~cm}^{-1}$ [7]. Có nghĩa là 20 dao động uốn này đã dịch chuyển rất nhiều về phía có bước sóng cao hớn. Điều này chỉ có thể giải thích là do ảnh hưởng của nhóm peroxid gây ra, chính nhóm này đã dùng năng lượng của hai vòng benzen để tạo ra hoạt tính rất mạnh, nó có thể là nguyên nhân gây ra tính kháng khuẩn, chống viêm mạnh BPO đã biết [6].

Trong vùng $1600-1400 \mathrm{~cm}^{-1}$ có dao động kéo dài của lõi phenyl $C C$ (gồm $C=C$ và $C-C$ ). Các dải kéo dài $\mathrm{C}=\mathrm{C}$ lần lượt là $1603,1597,1484,1448$ $\mathrm{cm}^{-1}$; liên kết $\mathrm{C}$ - C ở 1340, 1317, 1234 và 1220 $\mathrm{cm}^{-1}$. Do có sự liên kết chặt chẽ của nhóm peroxid với trực tiếp lõi phenyl, nên dao động của vòng kéo căng thường là theo đường chéo. Trừ một dải liên kết $\mathrm{C}=\mathrm{C}$, tất cả các dao động khác đều nằm dưới dải bước sóng theo như thông thường và toàn bộ dao động co giãn của liên kết C-C lệch khỏi vùng số sóng đặc trưng 
của nó. Hiện tượng các liên kết lệch khỏi vị trí số sóng là do các năng lượng trao đổi giữa hai vòng phenyl thông qua nhóm peroxid, đây có thể coi là đặc điểm tạo ra đặc tính của BPO. Vòng carbon (CCC) dao động trong và ngoài mặt phẳng tương ứng ở dải từ 200 đến $470 \mathrm{~cm}^{-1}$. Các vị trí dao động này của vòng carbon cũng không ở vùng số sóng thông thường và có cường độ yếu. Có thể cho rằng, do liên kết mạnh của nhóm peroxide nên kìm hãm sự dao động của vòng carbon.

Đối với các acid peroxid đều có dải hấp thụ yếu trong vùng $900-800 \mathrm{~cm}^{-1}$ do dao động kéo dài $\mathrm{O}-\mathrm{O}$. Acid peroxid cũng có hai dải mạnh ở $1840-1810 \mathrm{~cm}^{-1}$ và $1800-1770 \mathrm{~cm}^{-1}$ (béo bão hòa) do dao động kéo dài $\mathrm{C}=\mathrm{O}$ của chúng. Thông thường, cường độ của những dải tần ứng với liên kết $\mathrm{C}=\mathrm{O}$ này rất cao.

Ở đây, dao động kéo giãn O-O được tìm thấy với cường độ yếu ở tần số là $800 \mathrm{~cm}^{-1}$. Các dải uốn trong mă̆t phẳng và ngoài mặt phẳng tương ứng của $\mathrm{O}-\mathrm{O}$ lần lượt là 200 và $100 \mathrm{~cm}^{-1}$. Dải nằm ở phần cuối của vùng khảo sát, chủ yếu là liên kết lỏng lẻo và đặc tính đồng nhất cao. $\mathrm{C}=\mathrm{O}$ là phần chính của nhóm peroxid và dao động kéo dài của nó được quan sát ở 1782 và 1755 $\mathrm{cm}^{-1}$ trong phổ IR. Các số sóng này nằm lệch -50 $\mathrm{cm}^{-1}$ so với vùng số sóng tưng ứng với liên kết thông thường, tức là có thể gây ra biến đổi năng lượng trong phân tử để nó sinh ra đặc tính của hợp chất. Các chế độ uốn trong mặt phẳng và ngoài mặt phẳng $\mathrm{C}=\mathrm{O}$ lần lượt xuất hiện ở 460 và $445 \mathrm{~cm}^{-1}$ và 170 và $160 \mathrm{~cm}^{-1}$.

Các cực đại kéo dài $\mathrm{C}-\mathrm{O}$ xuất hiện ở 1176 và $1196 \mathrm{~cm}^{-1}$, liên kết này ở phần giữa của cấu trúc $\mathrm{BPO}$, nó có tác dụng gắn kết $\mathrm{O}-\mathrm{O}$ để giữ nó gần vòng benzen. Các chế độ uốn $\mathrm{C}-\mathrm{O}$ trong mặt phẳng lần lượt ở 350 và $325 \mathrm{~cm}^{-1}$ [8].

Bảng 4. Gán dao động đặc trưng phổ IR, Raman theo tính toán DFT và thực nghiệm.

\begin{tabular}{|c|c|c|c|c|c|}
\hline \multirow{2}{*}{$\begin{array}{l}\text { Thứ } \\
\text { tự }\end{array}$} & \multicolumn{2}{|c|}{ Thực nghiệm } & \multicolumn{2}{|c|}{ DFT/6-311G+(d,p) } & \multirow{2}{*}{ Gán dao động } \\
\hline & $\operatorname{IR}\left(\mathrm{cm}^{-1}\right)$ & $\operatorname{Raman}\left(\mathrm{cm}^{-1}\right)$ & IR $\left(\mathrm{cm}^{-1}\right)$ & $\operatorname{Raman}\left(\mathrm{cm}^{-1}\right)$ & \\
\hline 1 & - & $284 w$ & - & $268 w$ & $(\mathrm{CCC}) \mathrm{Y}$ \\
\hline 2 & $325 w$ & $350 w$ & $315 \mathrm{w}$ & $340 w$ & $(\mathrm{C}-\mathrm{O}) \delta$ \\
\hline 3 & - & $460 w$ & - & $445 \mathrm{w}$ & $(C=0) \delta$ \\
\hline 4 & - & $618 \mathrm{~s}$ & - & $628 \mathrm{w}$ & $(\mathrm{C}-\mathrm{H}) \mathrm{Y}$ \\
\hline 5 & - & $666 \mathrm{~m}$ & - & $684 w$ & $(\mathrm{C}-\mathrm{H}) \mathrm{Y}$ \\
\hline 6 & 692vs & - & $716 \mathrm{vs}$ & - & $(\mathrm{C}-\mathrm{H}) \mathrm{Y}$ \\
\hline 7 & $792 \mathrm{~s}$ & - & - & - & $(\mathrm{C}-\mathrm{H}) \mathrm{Y}$ \\
\hline 8 & - & $800 w$ & - & $812 w$ & $(0-0)$ u \\
\hline 9 & $846 s$ & $848 m$ & $860 \mathrm{~m}$ & $860 \mathrm{~m}$ & $(\mathrm{C}-\mathrm{H}) \overline{\mathrm{C}}$ \\
\hline 10 & $891 \mathrm{Vw}$ & $894 \mathrm{~s}$ & - & $940 \mathrm{~s}$ & $(\mathrm{C}-\mathrm{H}) \overline{\mathrm{C}}$ \\
\hline 11 & $941 w$ & - & - & - & $(\mathrm{C}-\mathrm{H}) \delta$ \\
\hline 12 & 993 vs & $1003 \mathrm{vs}$ & $1004 v s$ & $1020 \mathrm{vs}$ & $(\mathrm{C}-\mathrm{H}) \mathrm{\delta}$ \\
\hline 13 & $1035 s$ & $1025 \mathrm{~m}$ & - & $1036 \mathrm{~s}$ & $(\mathrm{C}-\mathrm{H}) \delta$ \\
\hline 14 & $1072 \mathrm{~m}$ & - & $1052 w$ & - & $(\mathrm{C}-\mathrm{H}) \delta$ \\
\hline 15 & - & - & $1108 \mathrm{vw}$ & - & $(\mathrm{C}-\mathrm{H}) \delta$ \\
\hline 16 & $1176 s$ & - & $1196 \mathrm{~m}$ & - & $(C-0) u$ \\
\hline 17 & $1220 \mathrm{vs}$ & $1234 v s$ & $1236 \mathrm{vs}$ & $1244 v s$ & $(C-C) U$ \\
\hline 18 & $1317 \mathrm{~s}$ & - & $1340 \mathrm{vw}$ & - & $(C-C) U$ \\
\hline 19 & $1448 \mathrm{vs}$ & - & $1484 \mathrm{vw}$ & - & $(C=C) U$ \\
\hline 20 & $1597 \mathrm{~s}$ & - & $1644 \mathrm{vw}$ & - & $(C=C) U$ \\
\hline 21 & $1755 \mathrm{vs}$ & - & 1812vs & - & $(\mathrm{C}=0) \mathrm{U}$ \\
\hline 22 & - & $1603 \mathrm{vs}$ & - & $1644 \mathrm{vs}$ & $(C=C) \cup$ \\
\hline 23 & $1782 \mathrm{~s}$ & $1778 \mathrm{vs}$ & $1836 \mathrm{~s}$ & $1836 \mathrm{vs}$ & $(\mathrm{C}=\mathrm{O}) \mathrm{U}$ \\
\hline
\end{tabular}

3.4. Tính chất nhiệt động học. Kết quả tính toán một vài đặc trưng về tính chất nhiệt động của BPO tại $\mathrm{T}=298,15 \mathrm{~K}, \mathrm{P}=1,00 \mathrm{~atm}$ là: tổng năng lượng nhiệt $(139,998 \mathrm{kcal} / \mathrm{mol})$, năng lượng dao động $(138,220 \mathrm{kcal} / \mathrm{mol})$, năng lượng dao động điểm Zero $(130,71093 \mathrm{kcal} / \mathrm{mol})$, nhiệt dung mol đẳng tích $C_{v}(56,004 \mathrm{cal} / \mathrm{mol} . \mathrm{K})$ và entropy $\mathrm{S}(129,071 \mathrm{cal} / \mathrm{mol} . \mathrm{K})$.

3.5. Tính chất hóa lý. Các tính chất hóa học liên quan đến cấu trúc phân tử và mức năng lượng biên phân tử của $\mathrm{BPO}$ được tính toán và toàn bộ các thông số được trình bày trong bảng 5 . 
Bảng 5. Bảng các thông số hóa lý của BPO.

\begin{tabular}{|c|c|}
\hline Thông số & $\begin{array}{c}\text { B3LYP 6- } \\
311 \mathrm{G}+(\mathrm{d}, \mathrm{p})\end{array}$ \\
\hline $\mathrm{E}_{\text {total }}(\mathrm{kcal} / \mathrm{mol})$ & $-840,38 .$. \\
\hline Еномо $(\mathrm{eV})$ & $-7,48558$ \\
\hline ELUMo(eV) & $-1,97337$ \\
\hline$\Delta$ Eномо-Luмо $(\mathrm{eV})$ & $-5,51221$ \\
\hline Độ cứng hóa học $\eta(\mathrm{eV})$ & 2,75611 \\
\hline Độ âm điện $\chi(\mathrm{eV})$ & $-4,72955$ \\
\hline Thế hóa học $\mu(\mathrm{eV})$ & 4,72955 \\
\hline Độ mềm hóa học $\sigma(1 / \mathrm{eV})$ & 0,36283 \\
\hline Chỉ số độ nhiêm điện $\omega(\mathrm{eV})$ & 4,058 \\
\hline
\end{tabular}

Độ rộng vùng cấm năng lượng của các obitan phân tử (HOMO và LUMO) của BPO được tính toán là $5,51221 \mathrm{eV}$ cho thấy sự ổn định hóa học cao của nó.

Độ âm điện và thế hóa học có cùng độ lớn là 4,72955 cho thây nếu có phản ứng thì BPO sẽ tóa nhiêt và có độ lớn xác định. Giá trị của thế năng hóa học khoảng 4,73 eV cho thãy các liên kết hóa học chặt chẽ và độ bền liên kết của electron cao. Ta biết độ âm điện vòng benzen khoảng 2,09 eV, còn kết quả tính cho BPO là lớn khoảng 4,73 eV, tức là có sự dịch chuyển năng lượng thông qua các obitan xen phủ nhau, điều đó được cho là do sự đối xứng của vòng benzen hai bên nhóm peroxide. Tính toán DFT còn cho chỉ số nhiễm điện của BPO có giá trị lớn là $\omega=$ 4,058 . Hình 6 cho biết orbital phân tử cao nhất (HOMO) và thấp nhất của BPO theo lý thuyết DFT.

b)

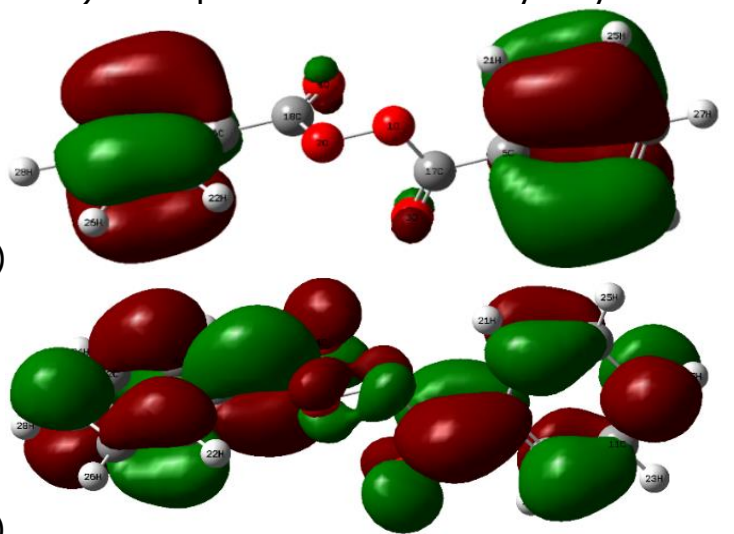

Hình 6. a) LUMO và b) HOMO của BPO theo tính toán DFT.

Theo động lực học, khi BPO được hấp thu vào da nó bị phân hủy tạo thành acid benzoic để loại bỏ Propionibacterium acnes và hình thành oxy mới để loại bỏ vi khuẩn yếm khí. Đồng thời, khi BPO ở trong mụn thì nó làm giảm đáng kể nồng độ acid béo và sự phát huỳnh quang của nang lông. Đăc tính kháng sinh manh cùa BPO trên có thể là do các đặc tính lý hóa được phân tích ở trên.

\section{KẾT LUÂNN}

Việc sử dụng phương pháp tính toán DFT đã tối ưu hóa vị trí và gắn số các nguyên tử trong phân tử BPO trong pha khí. Từ sự tối ưu cấu trúc, các tính toán đưa ra vài đặc trưng cho tính chất vật lý thường được sử dụng như khối lượng, diện tích bề mă̆t.

Kết hợp với phương pháp DFT thì các mode dao động đặc trưng của phổ Raman, IR đã được phân tích, chúng tôi cũng đã gán được các kiểu dao động này cho các đỉnh phổ thực nghiệm đặc trưng của BPO. Việc phân tích sự dịch chuyển lớn số sóng tương ứng với các liên kết đặc trưng có thể là nguyên nhân gây ra đặc tính kháng sinh của BPO.

Các tính chất nhiệt động và hóa lý của BPO cho thấy sự ổn định về tính chất vật lý và hóa hoc trong điều kiện tự nhiên. Các giá trị về tổng nắng lượng phân tử, năng lượng HUMO và LUMO, cũng như giá trị độ âm điện và thế hóa học đặc trưng cho tính chất hóa lý cũng cho thây BPO có đặc tính kháng sinh và chống viêm mạnh.

\section{TÀI LIẸU THAM KHẢO}

1. K. Okamoto et al.(2016), "In vitro antimicrobial activity of benzoyl peroxide against Propionibacterium acnes assessed by a novel susceptibility testing method," J. Infect. Chemother., 22 (6), pp. 426-429.

2. A. Nast et al.(2012), "European evidence-based (S3) guidelines for the treatment of acne," J. Eur. Acad. Dermatology Venereol., 26(1), pp. 1-29.

3. M. Sagransky, B. A. Yentzer, and S. R Feldman (2009), "Benzoyl peroxide: A review of its current use in the treatment of acne vulgaris," Expert Opin. Pharmacother., 10(15), pp. 2555-2562.

4. R. Anitha, M. Gunasekaran, S. S. Kumar, S. Athimoolam, and B. Sridhar (2015), "Single crystal XRD, vibrational and quantum chemical calculation of pharmaceutical drug paracetamol: A new synthesis form," Spectrochim. Acta - Part A Mol. Biomol. Spectrosc., 150, pp. 488-498.

5. R. Dennington, T. Keith, and J. Millam (2009), "GaussView, Version 5. J. Semichem Inc., Shawnee Mission."

6. R. Aarthi, S. Ramalingam, S. Periandy, and K. Senthil Kannan (2018), "Molecular structureassociated pharmacodynamic investigation on benzoyl peroxide using spectroscopic and quantum computational tools," J. Taibah Univ. Sci., 12(1), pp. 104-122.

7. S. Muthu, G. Ramachandran, and J. Uma Maheswari (2012), "Vibrational spectroscopic investigation on the structure of 2-ethylpyridine-4carbothioamide," Spectrochim. Acta - Part A Mol. Biomol. Spectrosc., 93, pp. 214-222.

8. J. S. Al-Otaibi (2018), "Molecular structure, spectroscopic (FT-IR, FT-Raman) and homo-lumo analyses of some acne vulgaris drugs," Rasayan J. Chem., 11(1), pp. 88-102. 